\title{
Iterative image reconstruction in elastic inhomogenous media with application to transcranial photoacoustic tomography
}

Joemini Poudel, Thomas P. Matthews, Kenji Mitsuhashi, Alejandro Garcia-Uribe, Lihong V. Wang, et al.

Joemini Poudel, Thomas P. Matthews, Kenji Mitsuhashi, Alejandro GarciaUribe, Lihong V. Wang, Mark A. Anastasio, "Iterative image reconstruction in elastic inhomogenous media with application to transcranial photoacoustic tomography," Proc. SPIE 10139, Medical Imaging 2017: Ultrasonic Imaging and Tomography, 101390C (13 March 2017); doi: 10.1117/12.2254141 


\title{
Iterative image reconstruction in elastic inhomogenous media with application to transcranial photoacoustic tomography
}

\author{
Joemini Poudel ${ }^{\mathrm{a}}$, Thomas P. Matthews ${ }^{\mathrm{a}}$, Kenji Mitsuhashi ${ }^{\mathrm{a}}$, Alejandro Garcia-Uribe ${ }^{\mathrm{a}}$, Lihong \\ V. Wang ${ }^{\mathrm{a}}$, and Mark A. Anastasio ${ }^{\mathrm{a}}$ \\ aDepartment of Biomedical Engineering, Washignton University in St. Louis, 1 Brookings Dr., \\ St. Louis, MO, USA 63130
}

\begin{abstract}
Photoacoustic computed tomography (PACT) is an emerging computed imaging modality that exploits optical contrast and ultrasonic detection principles to form images of the photoacoustically induced initial pressure distribution within tissue. The PACT reconstruction problem corresponds to a time-domain inverse source problem, where the initial pressure distribution is recovered from the measurements recorded on an aperture outside the support of the source. A major challenge in transcranial PACT brain imaging is to compensate for aberrations in the measured data due to the propagation of the photoacoustic wavefields through the skull. To properly account for these effects, a wave equation-based inversion method should be employed that can model the heterogeneous elastic properties of the medium. In this study, an iterative image reconstruction method for 3D transcranial PACT is developed based on the elastic wave equation. To accomplish this, a forward model based on a finite-difference time-domain discretization of the elastic wave equation is established. Subsequently, gradient-based methods are employed for computing penalized least squares estimates of the initial source distribution that produced the measured photoacoustic data. The developed reconstruction algorithm is validated and investigated through computer-simulation studies.
\end{abstract}

Keywords: Photoacoustic computed tomography,image reconstruction, transcranial imaging, elastic wave equation

\section{INTRODUCTION}

Photoacoustic computed tomography (PACT) is a noninvasive imaging modality that exploits the optical absorption contrast of tissue with the high spatial resolution of ultrasound imaging techniques. ${ }^{1,2}$ In PACT, a short laser pulse is used to irradiate a biological sample, upon which optical absorption by tissues results in emission of pressure waves through the photoacoustic effect. These pressure waves are subsequently detected by broadband ultrasound detectors. The image reconstruction problem in PACT is to estimate the spatially variant optical absorption properties of the tissue from the measured pressure waves. Such an image may be of great importance as there exists a strong correlation between electromagnetic absorption in tissue and the pathological condition of tissue. ${ }^{3-5}$

Transcranial brain imaging represents an important application that may benefit significantly by the development of PACT methods. Existing human brain imaging modalities include X-ray computed tomography $(\mathrm{CT})$, magnetic resonance imaging (MRI), positron emission tomography (PET), and ultrasonography. However, all these modalities suffer from significant shortcomings. X-ray CT, PET, and MRI are expensive and employ bulky and generally non-portable imaging equipment. Additionally, X-ray CT and PET employ ionizing radiation and are therefore not suitable for longitudinal studies and MRI-based methods are generally slow. Ultrasonography is an established portable pediatric brain imaging modality that can operate in near real-time, but its image quality degrades severely when employed after the closure of the fontanels. On the other hand, PACT can be implemented in near real-time, does not employ ionizing radiation, is much less costly than either MRI, PET or $\mathrm{X}$-ray CT, and can provide both anatomical and functional information.

Further author information: (Send correspondence to Mark A. Anastasio)

E-mail: anastasio@wustl.edu

Medical Imaging 2017: Ultrasonic Imaging and Tomography, edited by Neb Duric, Brecht Heyde, Proc. of SPIE Vol. 10139, 101390C · @ 2017 SPIE · CCC code: 1605-7422/17/\$18 $\cdot$ doi: 10.1117/12.2254141 
The majority of the currently available photoacoustic computed tomography (PACT) reconstruction algorithms are based on idealized imaging models that assume a lossless and acoustically homogeneous medium. ${ }^{6-9}$ However, in transcranial imaging applications, these assumptions are violated as induced photoacoustic wavefields undergo absorption, scattering and longitudinal-to-shear wave mode conversion due to the presence of the skull. ${ }^{10,11}$ Hence, the reconstructed images contain significant distortions and artifacts if the acoustic properties of the skull are not accounted for in the reconstruction algorithm. In order to model the acoustic heterogeneity of skull, some studies have developed subject-specific imaging models that use adjunct X-ray CT data to specify the skull morphology and composition. ${ }^{12}$ However, a limitation of such work is that it assumes a fluid medium and therefore assumes a simplified wave propagation model in which longitudinal-to-shear-wave mode conversion within the skull, which is an elastic solid, is negelected. ${ }^{13,14}$

To circumvent limitations of previous approaches, in this work an image reconstruction algorithm in transcranial PACT based on an elastic wave equation that describes an linear isotropic, lossy and heterogeneous medium is developed and investigated. The availability of massively parallel implementations of the matched forward and adjoint operator pair using multiple GPUs ${ }^{15}$ permits the application of iterative reconstruction algorithms that can model the imaging physics accurately as well as effectively mitigate image artifacts due to data incompleteness and noise. In this study, an iterative image reconstruction algorithm that seeks to minimize a penalized least squares (PLS) cost function was developed and investigated through computer-simulation studies.

\section{BACKGROUND}

Let the photoacoustically-induced stress tensor at location $\mathbf{r} \in \mathbb{R}^{3}$ and time $t \geq 0$ be defined as

$$
\boldsymbol{\sigma}(\mathbf{r}, t) \equiv\left[\begin{array}{ccc}
\boldsymbol{\sigma}^{11}(\mathbf{r}, t) & \boldsymbol{\sigma}^{12}(\mathbf{r}, t) & \boldsymbol{\sigma}^{13}(\mathbf{r}, t) \\
\boldsymbol{\sigma}^{21}(\mathbf{r}, t) & \boldsymbol{\sigma}^{22}(\mathbf{r}, t) & \boldsymbol{\sigma}^{23}(\mathbf{r}, t) \\
\boldsymbol{\sigma}^{31}(\mathbf{r}, t) & \boldsymbol{\sigma}^{32}(\mathbf{r}, t) & \boldsymbol{\sigma}^{33}(\mathbf{r}, t)
\end{array}\right],
$$

where $\boldsymbol{\sigma}^{i j}(\mathbf{r}, t)$ represents the stress in the $i^{\text {th }}$ direction acting on a plane perpendicular to the $j^{\text {th }}$ direction. Additionally, let $p_{0}(\mathbf{r})$ denote the photoacoustically-induced initial pressure distribution within the object, and $\dot{\mathbf{u}}(\mathbf{r}, t) \equiv\left(\dot{u}^{1}(\mathbf{r}, t), \dot{u}^{2}(\mathbf{r}, t), \dot{u}^{3}(\mathbf{r}, t)\right)$ represent the vector-valued acoustic particle velocity. Let $\rho(\mathbf{r})$ denote medium's density distribution and $\lambda(\mathbf{r}), \mu(\mathbf{r})$ represent the Lamé parameters that describe the full elastic tensor of the linear isotropic media. All functions in this work are assumed to be bounded and compactly supported. In a 3D heterogeneous linear isotropic elastic medium with a diffusive acoustic absorption coefficient $\alpha(\mathbf{r})$, the propagation of $\dot{\mathbf{u}}(\mathbf{r}, t)$ and $\boldsymbol{\sigma}(\mathbf{r}, t)$ can be modeled by the following two coupled equations: ${ }^{16,17}$

$$
\partial_{t} \dot{\mathbf{u}}(\mathbf{r}, t)+\alpha(\mathbf{r}) \dot{\mathbf{u}}(\mathbf{r}, t)=\frac{1}{\rho(\mathbf{r})}(\nabla \cdot \boldsymbol{\sigma}(\mathbf{r}, t))
$$

and

$$
\partial_{t} \boldsymbol{\sigma}(\mathbf{r}, t)=\lambda(\mathbf{r}) \operatorname{tr}(\nabla \dot{\mathbf{u}}(\mathbf{r}, t)) \mathbf{I}+\mu(\mathbf{r})\left(\nabla \dot{\mathbf{u}}(\mathbf{r}, t)+\nabla \dot{\mathbf{u}}(\mathbf{r}, t)^{T}\right),
$$

subject to the initial conditions

$$
\left.\boldsymbol{\sigma}_{0}(\mathbf{r}) \equiv \boldsymbol{\sigma}(\mathbf{r}, t)\right|_{t=0}=-\frac{1}{3} p_{0}(\mathbf{r}) \mathbf{I},\left.\quad \dot{\mathbf{u}}(\mathbf{r}, t)\right|_{t=0}=0 .
$$

Here, $\operatorname{tr}(\cdot)$ is the operator that calculates the trace of a matrix and $\mathbf{I} \in \mathbb{R}^{3 \times 3}$ is the identity matrix. In Eqn. $2 \mathrm{c}$, it has been assumed that the object function $p_{0}(\mathbf{r})$ is compactly supported in a fluid medium where the shear modulus $\mu(\mathbf{r})=0$. In transcranial PACT, this corresponds to the situation where the initial photoacoustic wavefield is produced within the soft tissue enclosed by the skull.

Consider that $p(\mathbf{r}, t) \equiv \operatorname{tr}(\boldsymbol{\sigma}(\mathbf{r}, t))$ be the pressure recorded outside the support of the object for $\mathbf{r} \in \mathrm{d} \Omega$ and $t \in[0, T]$, where $\mathrm{d} \Omega \subset \mathbb{R}^{3}$ denote a continous measurement aperture. In this case, the imaging model can be described as a continous-to-continous $(\mathrm{C}-\mathrm{C})$ mapping as:

$$
p(\mathbf{r}, t)=\mathcal{M H} p_{0}(\mathbf{r}),
$$




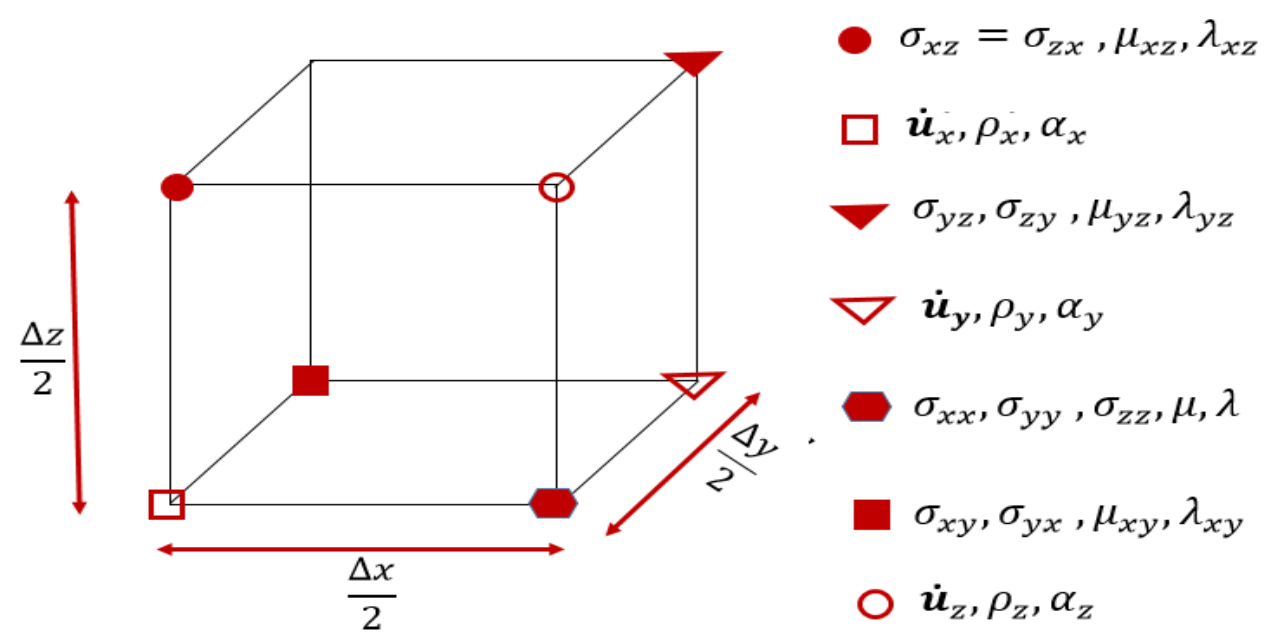

Figure 1: A staggered-grid FD cell with positions of the wavefield variables ${ }^{18}$

where $\mathcal{H}: \mathbb{L}^{2}(\Omega) \rightarrow \mathbb{L}^{2}(\Omega \times[0, T])$ is a linear operator that denotes the action of the wave equation given by Eqn. (2), $p(\mathbf{r}, t) \in \mathbb{L}^{2}(\mathrm{~d} \Omega \times[0, T])$ denotes the measured data function and the operator $\mathcal{M}$ is the restriction of $\mathcal{H}$ to $\mathrm{d} \Omega \times[0, T]$.

In practice the detected pressure wavefield is discretized spatially and temporally at specific transducer locations. Let $\mathbf{p} \in \mathbb{R}^{N_{r} L}$ denote the discretized pressure signal. Hence, a continous-to-discrete (C-D) imaging model can be generally expressed as

$$
[\mathbf{p}]_{k L+l}=\left.p(\mathbf{r}, t)\right|_{\mathbf{r}=\mathbf{r}_{0}^{k}, t=l \Delta^{t}}
$$

for $k=0,1,2, \ldots, N_{r}-1$ and $l=0,1,2, \ldots, L-1$. Here, $L$ is the total number of temporal samples, $\Delta^{t}$ is the temporal sampling interval, and the vectors $\mathbf{r}_{0}^{k} \in \mathbb{R}^{3}, k=0,1,2, \ldots, N_{r}-1$, represent the position vectors of the $N_{r}$ receivers on the aperture $\mathrm{d} \Omega$.

To obtain a discrete-to-discrete (D-D) imaging model for use in numerically simulating PA wavefield propagation, a finite-dimensional approximate representation of the object function $p_{0}(\mathbf{r})$ as well as the medium properties $\alpha(\mathbf{r}), \lambda(\mathbf{r}), \mu(\mathbf{r})$, and $\rho(\mathbf{r})$ needs to be introduced. The way in which the material parameters $\alpha(\mathbf{r}), \lambda(\mathbf{r}), \mu(\mathbf{r})$, and $\rho(\mathbf{r})$ as well as the object function $p_{0}(\mathbf{r})$ is discretized to form lexicographically ordered vector representations $\boldsymbol{\alpha}, \boldsymbol{\lambda}, \boldsymbol{\mu}, \boldsymbol{\rho}$ and $\mathbf{p}_{0} \in \mathbb{R}^{N \times 1}$, respectively, is dictated by the numerical method employed to solve the elastic wave equation. Here, $N$ represents the total number of grid points in the $3 \mathrm{D}$ grid. In this study, we employ a 4th-order staggered-grid finite difference time domain method (FDTD) to solve the initial value problem defined in Eqn. (2). For the staggered-grid finite difference (FD) scheme, the material properties, stress, and particle velocity functions are sampled at different points of the staggered FD cell of Fig. 1. Thus, given $\boldsymbol{\alpha}, \boldsymbol{\lambda}, \boldsymbol{\mu}, \boldsymbol{\rho}$ and $\mathbf{p}_{0}$, a D-D imaging model is given by,

$$
\mathbf{p}=\mathbb{M H} \mathbf{p}_{0},
$$

where $\mathbb{H} \in \mathbb{R}^{N L \times N}$ is the discrete approximation of the wave operator $\mathcal{H}$ that solves the initial value problem defined in Eqn. (2). Here, $\mathbb{M} \in \mathbb{R}^{N_{r} L \times N L}$ is a sampling matrix that models the PACT data acquisition process. For simplicity, we assume that the transducers are point-like in this study. When the receiver and grid point locations do not coincide, an interpolation method is required. Hence, the elements of $\mathbb{M}$ are chosen such that trilinear interpolation is performed. The goal of image reconstruction in a discrete setting is to determine an estimate of $\mathbf{p}_{0}$ by use the measured data $\mathbf{p}$.

\section{ITERATIVE IMAGE RECONSTRUCTION}

By use of the proposed D-D imaging model defined in Eqn. (5), a variety of image reconstruction algorithms can be employed for determining estimates of $\mathbf{p}_{0}$. In this work, we utilize an iterative PACT reconstruction 
algorithm that seeks to compute penalized least squares estimates by solving an optimization of the form

$$
\hat{\mathbf{p}_{0}}=\underset{\mathbf{p}_{0}}{\operatorname{argmin}}\left\|\mathbf{p}-\mathbb{M H} \mathbf{p}_{0}\right\|_{2}^{2}+\gamma \mathrm{R}\left(\mathbf{p}_{0}\right),
$$

where $\gamma$ is a regularization parameter and $\mathrm{R}\left(\mathbf{p}_{0}\right)$ is a regularizing penalty term. For this study, the total variance (TV) semi-norm penalty, given by

$$
\mathrm{R}\left(\mathbf{p}_{0}\right)=\left\|\mathbf{p}_{0}\right\|_{T V} \equiv \sum_{n=1}^{N}\left\{\left(\left[\mathbf{p}_{0}\right]_{n}-\left[\mathbf{p}_{0}\right]_{n 1^{-}}\right)^{2}+\left(\left[\mathbf{p}_{0}\right]_{n}-\left[\mathbf{p}_{0}\right]_{n 2^{-}}\right)^{2}+\left(\left[\mathbf{p}_{0}\right]_{n}-\left[\mathbf{p}_{0}\right]_{n 3^{-}}\right)^{2}\right\}^{\frac{1}{2}},
$$

was employed. Here, $[\boldsymbol{\theta}]_{n}$ denotes the $n^{t h}$ grid node, and $[\boldsymbol{\theta}]_{n 1^{-}},[\boldsymbol{\theta}]_{n 2^{-}}$, and $[\boldsymbol{\theta}]_{n 3^{-}}$are the neighboring nodes before the $n^{\text {th }}$ node along the first, second and third dimension, respectively. The fast iterative shrinkage/thresholding algorithm (FISTA $)^{19}$ was employed to solve the optimization problem in Eqn. (6).

\section{COMPUTER-SIMULATION STUDIES}

Computer-simulation studies were conducted in which the performance of the iterative reconstruction algorithm was compared with a canonical backprojection (BP) reconstruction algorithm. ${ }^{6,7}$ The BP method is based on a continuous PACT imaging model for an acoustically homogeneous fluid media and thus cannot account for the presence of skull.

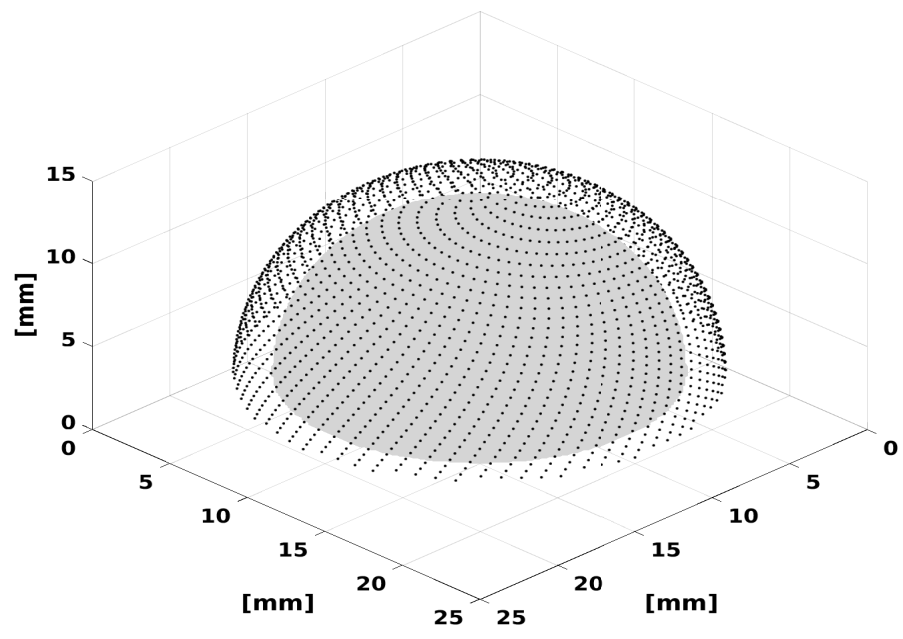

Figure 2: Figure showing the position of the hemispherical shell with respect to the transducer array.

\subsection{Methods}

A numerical 3D $(24 \mathrm{~mm} \times 24 \mathrm{~mm} \times 24 \mathrm{~mm})$ phantom, as shown in Fig. 3a, was employed for the initial pressure distribution in the computer simulation studies. The numerical phantom consisted of a point source placed inside a elastic isotropic lossy hemispherical shell $\left(\rho=1850 \frac{\mathrm{kg}}{\mathrm{m}^{3}}, \lambda=13.87 \mathrm{GPa}\right.$, and $\left.\mu=4.163 \mathrm{GPa}, \alpha=0.1 \frac{1}{\mu \mathrm{s}}\right)$, and the background medium was set to be water $\left(\rho=1000 \frac{\mathrm{kg}}{\mathrm{m}^{3}}, \lambda=2.25 \mathrm{GPa}\right.$, and $\left.\mu=0.0 \mathrm{GPa}, \alpha=0.0 \frac{1}{\mu \mathrm{s}}\right)$. The propagated pressure wavefield was recorded at 4400 transducers, uniformly placed on a hemispherical surface enclosing the shell. The location of hemispherical shell relative to the transducer array is shown in Figure 2. The 


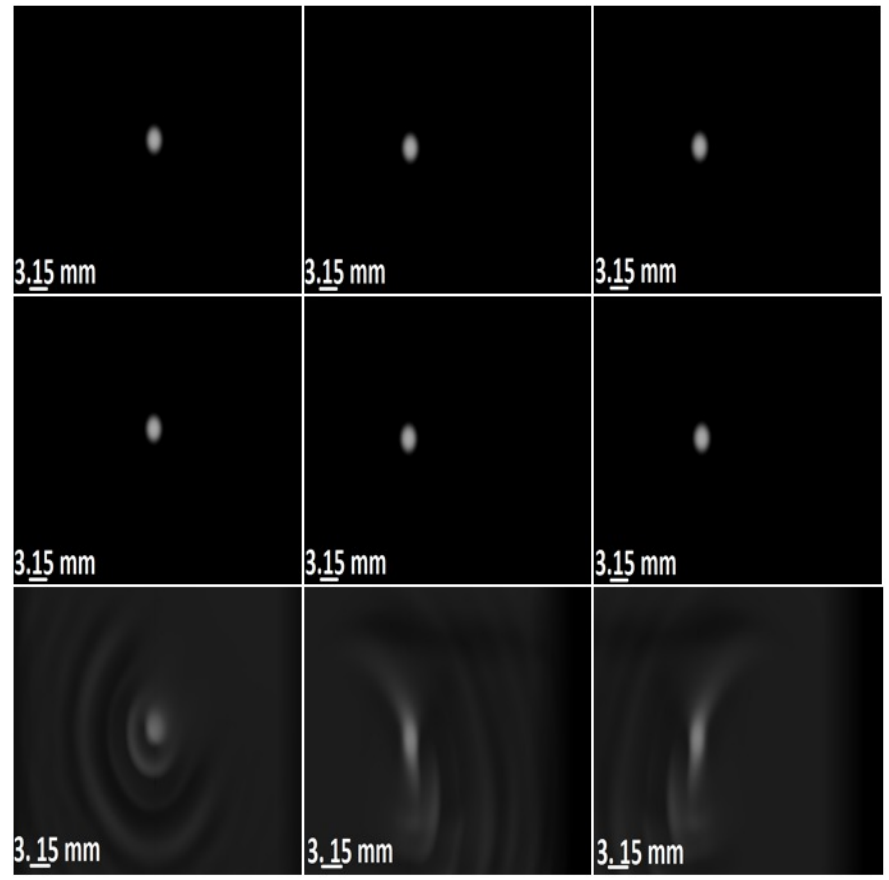

(a)

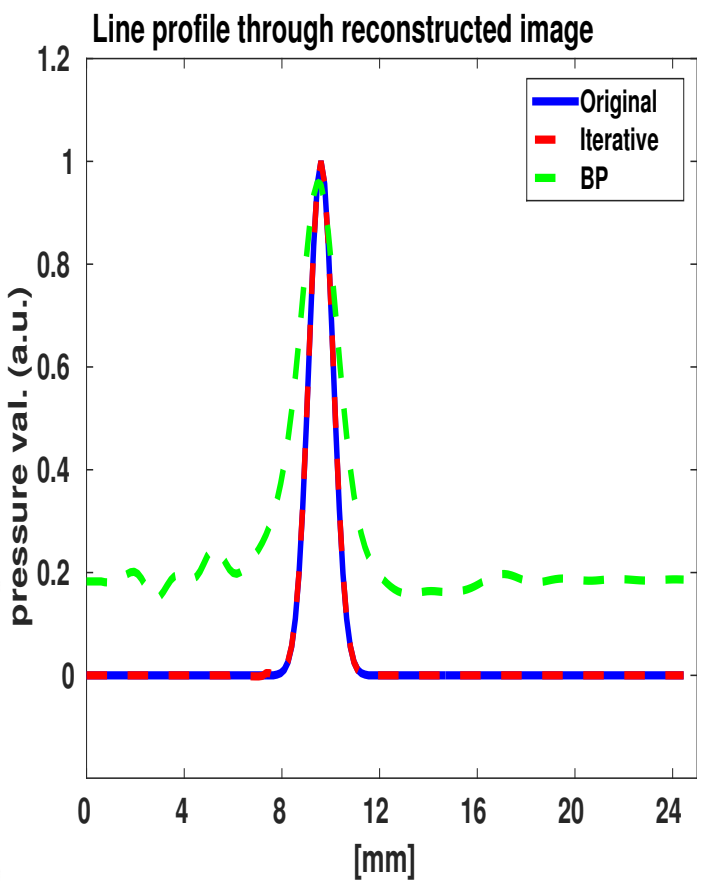

(b)

Figure 3: (a) The first row of images represents the maximum intensity projections of the initial pressure distribution along the $\mathrm{z}-, \mathrm{y}$ - and $\mathrm{x}$-axis, respectively. The second row of images represents the maximum intensity projections of the images reconstructed using the iterative algorithm along the $\mathrm{z}^{-}, \mathrm{y}-$ and $\mathrm{x}$-axis, respectively. The third row of images represents the maximum intensity projections of the images reconstructed using the BP method along the z-, y- and x-axis, respectively. All images are shown in a grayscale window of $[0,1]$. (b) The line profiles along the z-axis through the phantom and the reconstructed initial pressure distributions.

simulated pressure data at each transducer consisted of 1600 temporal samples that were sampled at a frequency of $200 \mathrm{MHz}$.

To study effectiveness of the proposed iterative algorithm in 3D transcranial PACT, we conducted noninverse crime computer-simulation studies, where different discretization strategies were employed to generate the measured data and to reconstruct the initial pressure distribution. ${ }^{20}$ The forward data was generated using an uniform grid of size $0.075 \mathrm{~mm}$ and was corrupted with white gaussian noise before applying the reconstruction algorithm. The iterative reconsturction algorithm was applied to the noisy forward data using a grid size of $0.15 \mathrm{~mm}$.

\subsection{Results}

The reconstructed images for the computer simulation study are shown in Fig. 3a. Images were obtained by use of the iterative reconstruction algorithm with a regularization parameter of $\gamma=0.01$. The reconstructed images produced by use of the iterative algorithm as wll as the BP algorithm are shown in Fig. 3a. In both cases, the results were displayed as maximum intensity projection (MIP) images along three mutually perpendicular directions. These results demonstrate that iterative algorithm can more effectively mitigate skull-induced image distortions than can the BP algorithm. The image reconstructed using the iterative algorithm possesses a much cleaner background and contains far fewer artifacts than the image reconstructed using the BP algorithm. This can also be seen quantitatively in the line profile shown in Fig. 3b. 


\section{CONCLUSION}

We demonstrated a full-wave approach to iterative image reconstruction in PACT with acoustically heterogenous elastic isotropic lossy media. The developed reconstruction methodology was investigated by use of computersimulation studies. The results demonstrated that the reconstruction methodology can effectively mitigate image artifacts induced by presence of elastic isotropic media. The proposed image reconstruction method could greatly improve the accuracy of reconstructed images for transcranial PACT applications.

\section{ACKNOWLEDGMENTS}

This work was supported in part by NIH awards EB01696301, 5T32EB01485505 and NSF award DMS1614305.

\section{REFERENCES}

[1] Kruger, R., Liu, P., and Appledorn, C., "Photoacoustic ultrasound (paus) reconstruction tomography," Med. Phys. 22, 605-1609 (1995).

[2] Kruger, R., Reinecke, D., and Kruger, G., "Thermoacoustic computed tomography- technical considerations," Med. Phys. 26, 1832-1837 (1999).

[3] Jones, W., Jirtle, R., Rafal, M., and Schaeffer, D., "Microwave power absorption differences between normal and malignant tissue," Radiat. Oncol., Biol., Phys., 6, 681-687 (1980).

[4] Cheong, W., Jirtle, R., Rafal, M., and Schaeffer, D., "Microwave power absorption differences between normal and malignant tissue," IEEE J. Quantum Elect. 26(12), 1086-1099 (2003).

[5] Xu, Z., Zhu, Q., and Wang, L. V., "In vivo photoacoustic tomography of mouse cerebral edema induced by cold injury," J. Biomed. Opt. 16(6), 066020 (2011).

[6] Kunyansky, L. A., "Explicit inversion formulae for the spherical mean radon transform," Inverse Problems 23, 373-383 (2007).

[7] Finch, D., Patch, S. K., and Rakesh, "Determining a function from its mean values over a family of spheres," SIAM Journal on Mathematical Analysis 35(5), 1213-1240 (2004).

[8] Finch, D., Haltmeier, M., and Rakesh, "Inversion of spherical means and the wave equation in even dimensions," SIAM Journal on Applied Mathematics 68(2), 392-412 (2007).

[9] Burgholzer, P., Bauer-Marshallinger, J., Grn, H., Haltmeier, M., and Paltauf, G., "Temporal back-projection algorithms for photoacoustic tomography with integrating line detectors," Inverse Problems 23, S65-S78 (Nov 2007).

[10] Jin, X., Li, C., Pan, X., and Wang, L., "Effects of acoustic heterogeneities on transcranial brain imaging with microwave-induced thermoacoustic tomography," Med. Phys. 35(7), 3205-3214 (2008).

[11] White, P., Clement, G., and Hynynen, K., "Longitudinal and shear mode ultrasound propagation in human skull bone," Ultrasound in medicine $\mathcal{E}$ biology 32(7), 1085-1096 (2006).

[12] Huang, C., Nie, L., Schoonover, R. W., Guo, Z., Schirra, C. O., Anastasio, M. A., and Wang, L. V., "Aberration correction for transcranial photoacoustic tomography of primates employing adjunct image data," Journal of Biomedical Optics 17(6), 066016-1-066016-8 (2012).

[13] Schoonover, R. W., Wang, L. V., and Anastasio, M. A., "Numerical investigation of the effects of shear waves in transcranial photoacoustic tomography with a planar geometry," Journal of Biomedical Optics 17(6), 061215-1-061215-11 (2012).

[14] Schoonover, R. W. and Anastasio, M. A., "Compensation of shear waves in photoacoustic tomography with layered acoustic media," JOSA A 28(10), 2091-2099 (2011).

[15] Mitsuhashi, K., Poudel, J., Matthews, T. P., Garcia-Uribe, A., Wang, L. V., and Anastasio, M. A., "A forward-adjoint operator pair based on the elastic wave equation for use in transcranial photoacoustic computed tomography," (2016). unpublished.

[16] Boore, D. M., "Finite-difference methods for seismic wave propagation in heterogeneous materials," in [Methods in Computational Physics], Bolt, B., ed., Academic Press, New York (1972).

[17] Virieux, J., "P-SV wave propagation in heterogeneous media: Velocity-stress finite-difference method," Geophysics 51, 889-901 (Apr 1986). 
[18] Moczo, P., Kristek, J., Galis, M., Pazak, P., and Balazovjech, M., "The finite-difference and finite-element modeling of seismic wave propagation and earthquake motion," acta physica slovaca 57(2), 177-406 (2007).

[19] Beck, A. and Teboulle, M., "Fast gradient-based algorithms for constrained total variation image denoising and deblurring problems," IEEE Trans. Image Process 18(11), 2419-2434 (2009).

[20] Colton, D. and Kress, R., [Inverse Acoustic and Electromagnetic Scattering Theory], Springer, New York, 2nd ed. (2013). 